Annales Geophysicae (2001) 19: 311-320 (C) European Geophysical Society 2001

Santes

\title{
Compressional Pc5 type pulsations in the morningside plasma sheet
}

\author{
A. Vaivads ${ }^{1,}$, W. Baumjohann ${ }^{1}$, G. Haerendel ${ }^{1}$, R. Nakamura ${ }^{1}$, H. Kucharek ${ }^{1}$, B. Klecker ${ }^{1}$, M. R. Lessard ${ }^{2}$, L. M. \\ Kistler $^{3}$, T. Mukai ${ }^{4}$, and A. Nishida ${ }^{4}$ \\ ${ }^{1}$ Max-Planck-Institut für extraterrestrische Physik, Garching, Germany \\ ${ }^{2}$ Dartmouth College, Thayer School of Engineering, Hanover, USA \\ ${ }^{3}$ Space Science Center, University of New Hampshire, USA \\ ${ }^{4}$ Institute for Space and Astronautical Science, Sagamihara, Japan \\ *Now at: Swedish Institute of Space Physics, Uppsala Division, Box 537, SE-75121 Uppsala, Sweden
}

Received: 13 June 2000 - Revised: 24 January 2001 - Accepted: 30 January 2001

\begin{abstract}
We study compressional pulsations in Pc5 frequency range observed in the dawn-side at distances of about $10 R_{E}$, close to the magnetic equator. We use data obtained during two events of conjunctions between Equator-S and Geotail: 1000-1700 UT on 9 March 1998, and 0200-0600 UT on 25 April 1998. In both events, pulsations are observed after substorm activity. The pulsations are antisymmetric with respect to the equatorial plane (even mode), and move eastward with phase velocity close to plasma velocity. The pulsations tend to be pressure balanced. We also discuss possible generation mechanisms of the pulsations.
\end{abstract}

Key words. Magnetospheric physics (magnetospheric configuration and dynamics; MHD waves and instabilities; plasma sheet)

\section{Introduction}

Strong compressional Pc5 pulsations, i.e. variations in the magnetic field magnitude with periods of typically about 10 minutes, are commonly observed in the outer magnetosphere at low geomagnetic latitudes. The Equator-S and Geotail satellites are well suited for studies of these pulsations because their orbits are close to the equatorial plane and cover large $L$ distances. Equator-S, with its apogee at about $11.5 R_{E}$, stays particularly long in regions where the compressional pulsations are observed. We use data from two conjunctions between Equator-S and Geotail to study these pulsations.

Compressional Pc5 pulsations are most often observed at large $L$ distances, $L \geq 8$, and there is a vast amount of data and studies related to this subject. Nevertheless, fundamental issues related to their generation, structure, lifetime, relation to observed shear waves, and relation to ground observations are still not settled. The term "compressional Pc5 pulsations" still reflects primarily the time scale of the variations in the

Correspondence to: A. Vaivads (andris@irfu.se) magnetic field magnitude, and not so much the physics behind it. Even sometimes the time scale can be outside of the Pc5 range (150-600 s), as defined by Jacobs et al. (1964).

Statistical studies of spacecraft data (Anderson et al., 1990; Hedgecock, 1976; Takahashi et al., 1990; Zhu and Kivelson, 1991; Lessard et al., 1999) show that compressional Pc5 pulsations are dominant pulsations at large distances, $L \geq 8$, but are less common at synchronous orbit. They have highest occurrence rates in the morning and afternoon sectors. Waves are observed close to the magnetic equator (within about $20^{\circ}$ ). The amplitude of pulsations correlate with plasma $\beta$ (a ratio between thermal and magnetic pressures); the highest amplitude waves are in regions of high- $\beta$ plasma (amplitudes can be as high as half of the ambient magnetic field). Magnetic and thermal pressure in pulsations are primarily in antiphase and nearly balance each other. Pulsations are polarized close to the meridian plane with comparable compressional and transverse components. The oscillations in inclination are such that low inclination corresponds to a high magnetic field.

In several cases, it has been possible to estimate the transverse size of pulsations either using two satellites or the finite gyroradius method (Takahashi et al., 1985; Lin et al., 1988; Zhu and Kivelson, 1994). The typical azimuthal size is $0.5-5 R_{E}$. The radial scale size is usually larger than the azimuthal. The magnetic equator is a node for the compressional component and antinode for the transverse component, i.e. the pulsations are antisymmetric with respect to the equator (even mode) (Takahashi et al., 1987; Zhu and Kivelson, 1994).

There are several explanations for the development of compressional Pc5 pulsations. Observations indicate that the mirror effect is dominating the response of the particles (Baumjohann et al., 1987; Zhu and Kivelson, 1994), and therefore, the phase velocity of the pulsations must have typical values that are much smaller than the thermal velocity of ions. It has been proposed that the pulsations are drifting plasma clumps or slow magnetosonic mode (Sonnerup, 1969) or drift mirror mode (Hasegawa, 1969). Later modifications in the drift 
mirror mode have been introduced, taking into account magnetic field curvature and coupling to compressional and shear Alfvén waves (Chen and Hasegawa, 1991, 1994; Cheng and Qian, 1994). There are also suggestions that compressional pulsations can be global modes of the magnetosphere (Kivelson et al., 1984).

Several studies have analyzed the phase velocity of compressional pulsations (e.g., Takahashi et al., 1985; Baumjohann et al., 1987). Most of these studies show that pulsations in the afternoon sector and on the day-side propagate westward. Only few studies show that compressional pulsations in the morning sector propagate eastward (e.g., Takahashi et al., 1987; Haerendel et al., 1999). In which direction compressional pulsations propagate in the plasma reference frame is still an open question.

The following generation mechanisms have been suggested for the compressional pulsations: plasma anisotropy (Hasegawa, 1969), pressure gradient, together with gradient and curvature in ambient magnetic field (Cheng and Qian, 1994; Chen and Hasegawa, 1991), and bounce-resonant particles (Southwood, 1976). Most of these mechanisms predict that in a high- $\beta$ plasma, the mode that will be generated first is antisymmetric with respect to the magnetic equator. There are also suggestions that compressional pulsations are generated through Kelvin-Helmholtz instability at the boundary between the central plasma sheet and the low latitude boundary layer (Rostoker and Samson, 1984), or within the regions of high velocity shears associated to the low- $m$ shear Alfvén waves (Allan and Wright, 1997).

This article is a continuation of the study done by Haerendel et al. (1999). In this article we study two compressional Pc5-type pulsation events on 9 March 1998 and 25 April 1998. Simultaneous Geotail and Equator-S conjunctions during these events, with satellites being on opposite sides of the magnetic equator, allow a detailed study of the structure of the pulsations. In particular, we compare the phase velocity of the pulsations with the plasma velocity.

We use data from the Equator-S magnetic field experiment (Fornacon et al., 1999) and moment data from the ion composition instrument (Kistler et al., 1999). From Geotail we use 12-sec averaged data from the Geotail magnetic field (Kokubun et al., 1994) and low energy particle experiment (Mukai et al., 1994).

\section{General event descriptions}

\subsection{The 9 March 1998 event}

Figure 1 shows the location of the Equator-S and Geotail satellites and their measurements of total, plasma, and magnetic pressure during the 9 March 1998 event. During this period both satellites are located in the dawn-sector close to, but on opposite sides of the geomagnetic equatorial plane. In comparison to Geotail, Equator-S moves more slowly and stays longer in the region. Both satellites observe compressional pulsations for many hours. Geotail ceases to see pul-
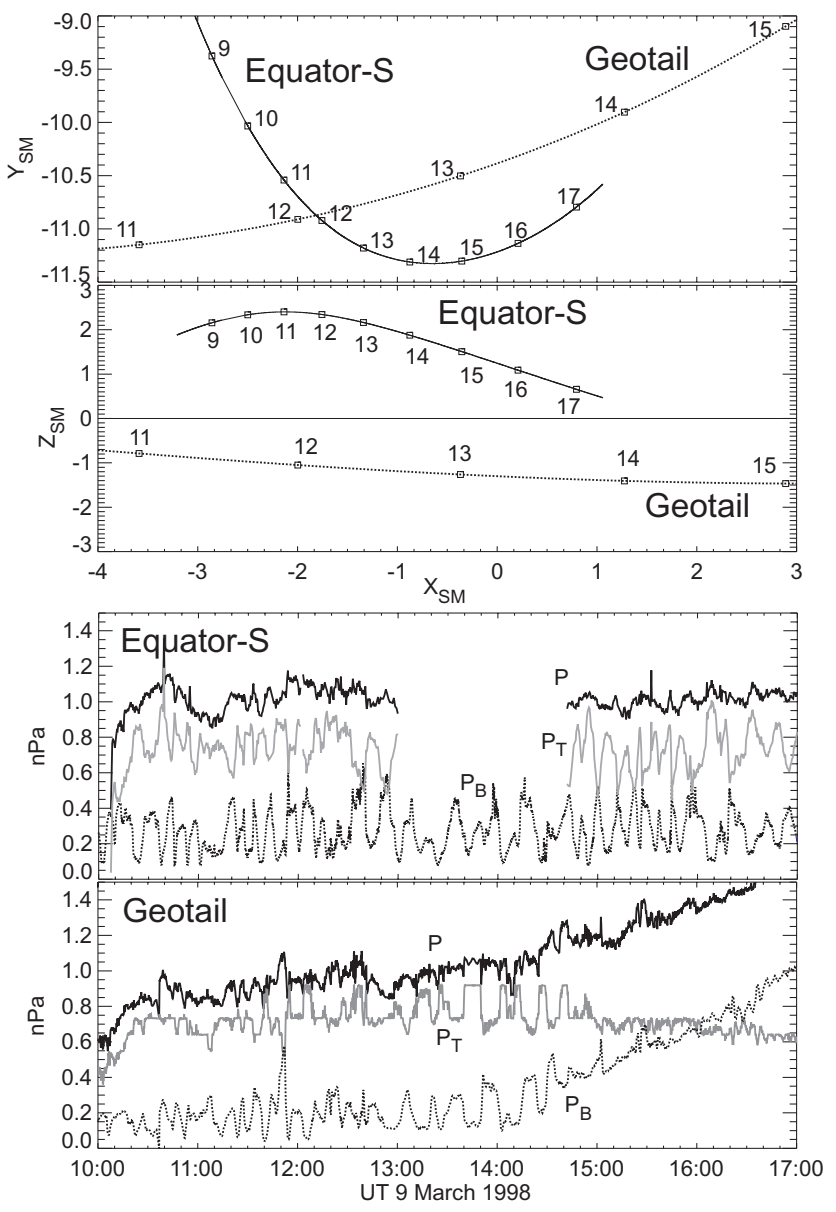

Fig. 1. The location of Equator-S and Geotail during event of 9 March 1998. Also shown are the total $P$, thermal $P_{T}$, and magnetic $P_{B}$ pressure measured by both satellites. The gap in the Equator-S data of the plasma pressure, between $\sim 1300$ UT and $\sim 1430$ UT, comes from a gap in the telemetry of the Ion Composition Instrument.

sations after $1500 \mathrm{UT}$, as it enters deeper into the magnetosphere. Equator-S observes pulsations until 1735 UT, when data transmission ends.

9 March 1998 was a moderately quiet day with AE index values below $200 \mathrm{nT}$. The IMF turned southward $\left(B_{z} \approx\right.$ $-3 \mathrm{nT}, B_{y} \approx 5 \mathrm{nT}$ ) at about $0700 \mathrm{UT}$. At $0820 \mathrm{UT}$ there is the first onset of a substorm followed by later substorms (or intensifications) at 0925 UT, 1010 UT, 1100 UT. Geotail EPIC observes injection of energetic electrons at $0830 \mathrm{UT}$ and energetic ions at about 0900 UT (not shown). Compressional pulsations start to appear with the energetic electrons. However, they obtain their maximum amplitude after the arrival of the energetic ions. Geotail also observes compressional pulsations several hours before the substorm (there are no data from Equator-S). Ground magnetometers show Pc5 and Ps6type pulsations in association with substorms (not shown).

In Fig. 2 and Fig. 3 we show more detailed plasma and magnetic field data from both Equator-S and Geotail during the time period around their conjunction. The conjunction 


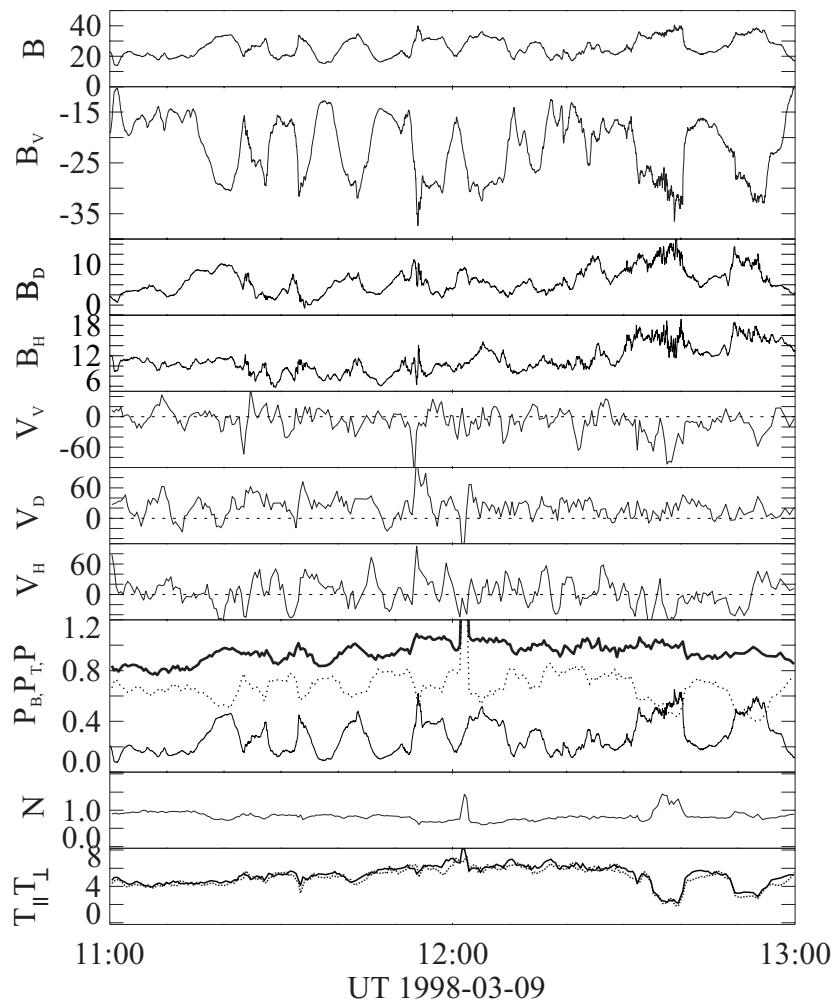

Fig. 2. Equator-S data for the time period around the EquatorS/Geotail conjunction on 9 March 1998. Data are presented in $V D H$ (radial $S M$ ) coordinates, where $V$ is radially outwards, $H$ is antiparallel to the direction of magnetic dipole and $D$ closes the system (approximately eastward). The different panels show: (a) the magnetic field magnitude, (b-d) $V, D$, and $H$ components of the magnetic field, (e-g) $V, D$, and $H$ components of the plasma velocity, (h) total, plasma and magnetic field pressure, (i) plasma density, (j) plasma temperature parallel (dotted) and perpendicular (solid) to the ambient magnetic field.

is at $\sim 1215$ UT. From Fig. 2 we see that the magnetic field at the Equator-S is dominated by the radial component. The azimuthal component of the velocity shows average sunward convection of the order of $20 \mathrm{~km} / \mathrm{s}$. We will discuss the correlation between velocity and magnetic field components in the next section. In the compressional pulsations that EquatorS observes between 1230 UT and 1300 UT, the temperature and density anticorrelates, while it correlates during the other time periods. This can indicate that between 1230 UT and 1300 UT Equator-S enters into the low-latitude boundary layer where an anticorrelation between temperature and density is common. Geotail, around the conjunction, is closer to the magnetic equator than Equator-S. The data confirm this; the magnetic field is not dominated by the radial component, even though the magnetic field variations are still largest in the radial component. In addition, Geotail is on closer Lshells than the Equator-S because the correlation between density and temperature indicates that Geotail does not enter the low-latitude boundary layer (except maybe for a few minutes, around 1240 UT). Similar to Equator-S, the plasma

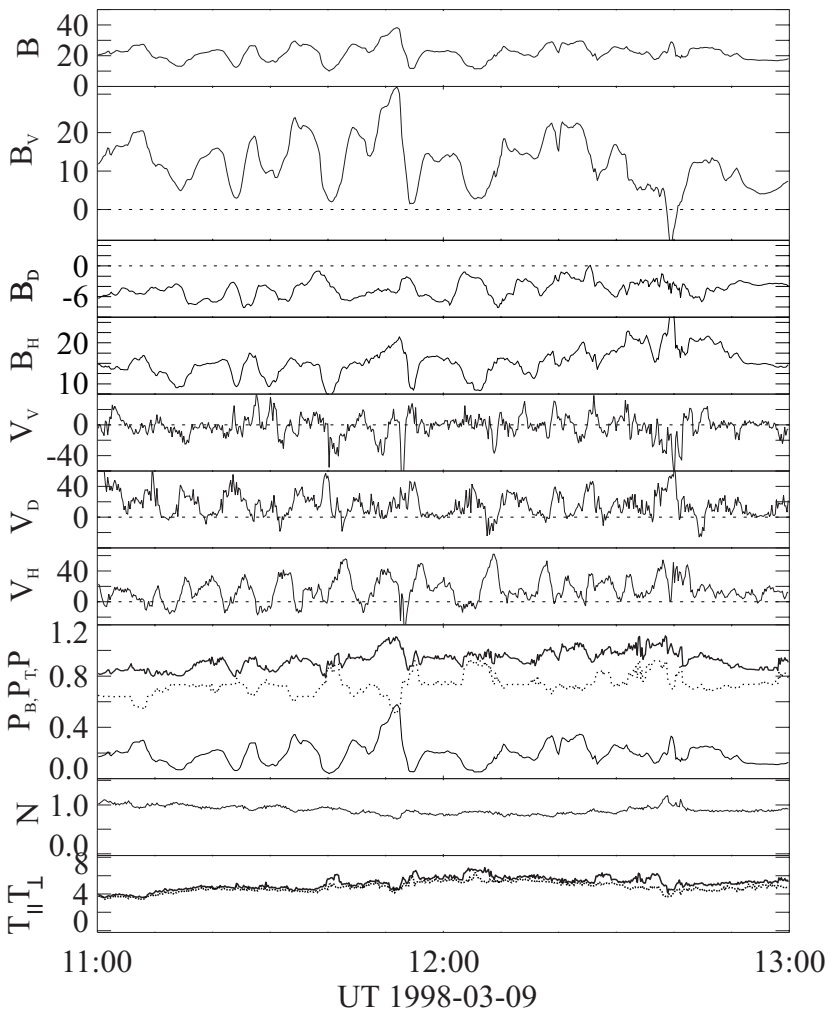

Fig. 3. Geotail data for the time period around the EquatorS/Geotail conjunction on 9 March 1998. Panels are the same as in Fig. 2.

velocity of Geotail shows an azimuthal convection sunward of about $20 \mathrm{~km} / \mathrm{s}$. Thus, both satellites are located within the plasma sheet.

\subsection{The 25 April 1998 event}

Figure 4 shows the location of the Equator-S and Geotail satellites and their measurements of total, plasma, and magnetic pressure during 25 April 1998 event. During this period both satellites are located in the early morning sector close to, but on opposite sides of the geomagnetic equatorial plane. Both satellites observe compressional pulsations between about 0320 UT and 0520 UT.

25 April 1998 was a geomagnetically active day with the AE index values reaching over 500 nT. Between 0200 UT and $0300 \mathrm{UT}$, one hour before the pulsation observations, there is a substorm with three intensifications. Nakamura et al. (1999) gives a thorough description of this substorm. The $D_{\text {st }}$ index indicates that this event occurs during the recovery phase of a magnetic storm.

\section{Data from Equator-S and Geotail}

\subsection{Magnetic field}

During both conjunctions the satellites are on opposite sides of the geomagnetic equator. This configuration was used by 

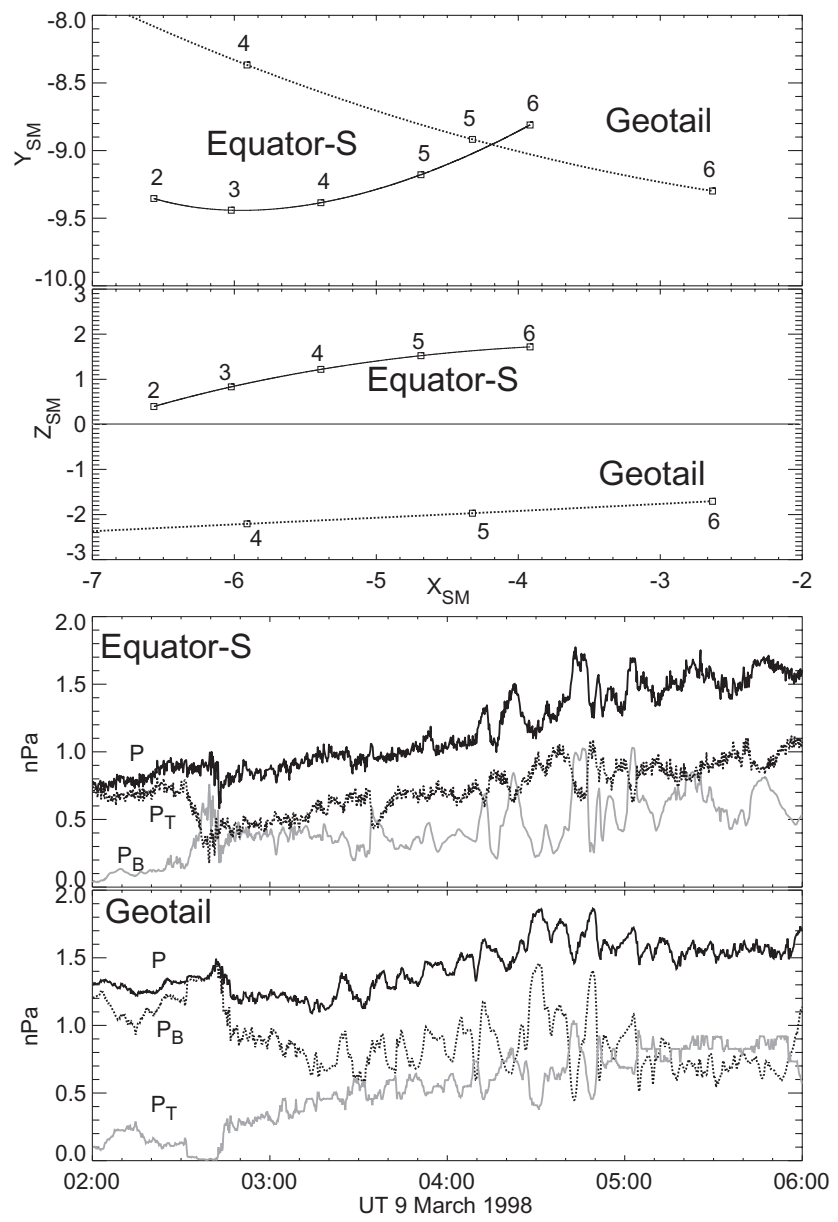

Fig. 4. The location of Equator-S and Geotail during event of 25 April 1998. Also shown are the total $P$, thermal $P_{T}$, and magnetic $P_{B}$ pressure measured by both satellites.

Haerendel et al. (1999) (see their Fig. 10) to show that the compressional pulsations are antisymmetric with respect to the equator (second harmonic type). In addition, the conjunctions allowed Haerendel et al. (1999) to estimate the azimuthal phase velocity and the azimuthal size of pulsations. Both for 9 March 1998 and 25 April 1998, the azimuthal size is about 10 degrees, which corresponds to m-values of about 35 . The azimuthal phase velocity values in SM coordinates are about $20 \mathrm{~km} / \mathrm{s}$ in the eastward direction, which is the direction of the plasma convection. Later we will analyze more thoroughly the propagation of compressional variations.

In Fig. 5, we sketch the antisymmetric standing mode as seen when looking from the dawn side towards the Earth and from the tail towards the sun. Similar sketches can be seen in earlier studies. For a 3D-sketch of the spatial structure of compressional pulsations, see Fig. 11 in Haerendel et al. (1999). For a similar 2D-sketch, see Fig. 8 in Takahashi et al. (1987a) or, for Jupiter's magnetosphere, Fig. 15 in Khurana and Kivelson (1989). For an example of a numerical solution to the structure of an antisymmetric drift mirror-ballooning mode, see Fig. 9 in Cheng and Qian (1994).

To compare the sketch of Fig. 5 with the data, we plot

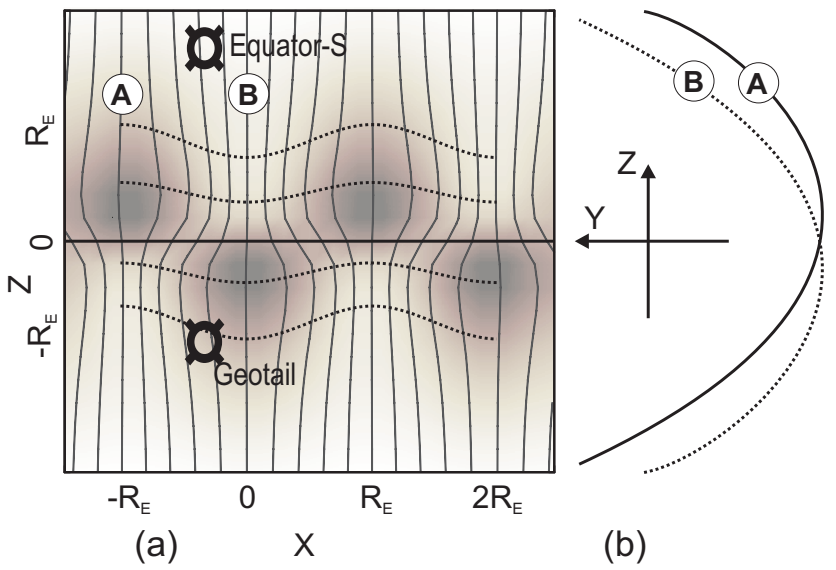

Fig. 5. Schematic illustration of the antisymmetric standing mode as seen from (a) the dawn-side towards the Earth and (b) from the tail-side towards the sun. Solid lines show magnetic field lines, shaded regions show high- $\beta$ plasma. Dotted lines show where surfaces of constant latitude cross the magnetic field lines. The position of Equator-S and Geotail satellites are also marked.

in Fig. 6 band-pass filtered data, $180 \mathrm{~s}<T<1800 \mathrm{~s}$, of Equator-S and Geotail in the mean field coordinates. We choose the time interval that includes the conjunction and the period before it. For almost the whole time interval, the polarization of the magnetic field observed by the satellites is consistent with the antisymmetric wave structure, as shown in Fig. 5, that does not change in time, but only passes from left to right (sunward or eastward motion). There are several observations that support it. At the conjunction, the magnetic field magnitudes seen by both satellites anticorrelate. Equator-S (which is farther away from the magnetic equator) shows that the variations in the magnetic field are mainly in the field aligned component; there is a small radial component that anticorrelates with the field aligned component. Geotail is closer to the magnetic equator and shows that the radial component is comparable to the field aligned component. Also consistent with the model is the phase lag of the azimuthal component, with respect to the field aligned component; at Equator-S, after $\sim 1130 \mathrm{UT}$, it is $-90^{\circ}$ and at Geotail, it is $90^{\circ}$.

The models of compressional Pc5 pulsations usually do not discuss the velocity components, except in a few cases, interpreting them as eventual $\boldsymbol{E} \times \boldsymbol{B}$ drifts. One thing to note is that, in general, the radial velocity component at both satellites lag in phase by $\sim 90^{\circ}$ the field aligned component of the magnetic field. We will speculate that this velocity component can be dominated by a diamagnetic drift. In this case the phase relationship is consistent with the model of Fig. 5. There are no clear phase relationships between the azimuthal velocity component and the magnetic field. At the same time, it seems that azimuthal velocities at both satellites are approximately in phase. This can indicate that part of the variations in the azimuthal velocity component are associated to some kind of large-scale azimuthal oscillations of 

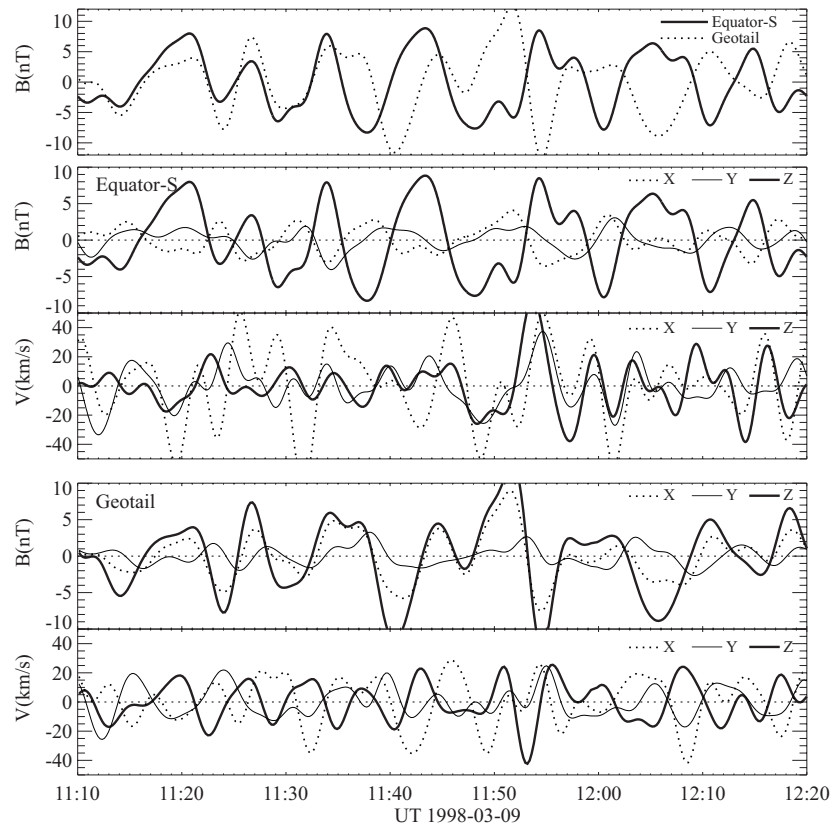

Fig. 6. The magnetic field and plasma velocity measured by Equator-S and Geotail, band-pass filtered $180 \mathrm{~s}<T<1800 \mathrm{~s}$ and plotted in the mean field coordinates. $Z$ - along the mean field direction, $X$ - perpendicular to the mean field in the meridional plane (positive outward), $Y$-closes the system (approximately eastward).

the plasma sheet. Finally, the field aligned velocity component tends to be in antiphase, with the radial velocity component at Geotail (no such phase relation is seen at Equator-S). There is no obvious explanation for this phase relationship, but Haerendel et al. (1999) has suggested that this is associated with plasma being sloshed along the magnetic field lines.

\subsection{Pressure balance}

In previous studies it has been shown that in compressional pulsations, magnetic and thermal pressure (the component perpendicular to the ambient magnetic field) tend to be in antiphase and cancel out each other (Zhu and Kivelson, 1994). Direct inspection of our data verifies this also in our case. Figure 1 and Fig. 4 show the measurements of the magnetic and thermal pressure. For Equator-S we plotted the pressure perpendicular to the ambient magnetic field summed over $\mathrm{H}^{+}$ and $\mathrm{O}^{+}$ions. Geotail cannot distinguish different ion species. The variations in the total pressure are small in comparison to the variations in magnetic and thermal pressure during 9 March 1998. Thus, pressure balance is approximately satisfied for the pulsations of this event. During 25 April 1998, the variations in total pressure are much larger than the variations in thermal pressure, which would indicate that pressure balance is not satisfied. However, during 25 April 1998, a large part of the ion population has energies above $40 \mathrm{keV}$, which is above the upper limit of ion instruments used in the calculation of plotted pressures (the peak of differential energy flux for protons seems to be close to $40 \mathrm{keV}$ ). Thus, the

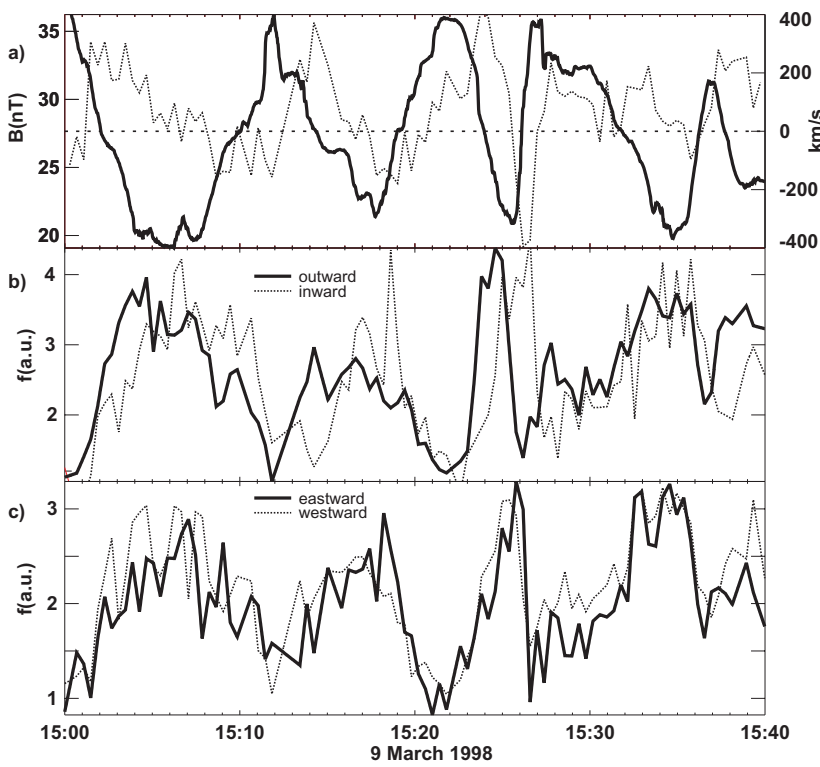

Fig. 7. Finite gyroradius method for 1500-1540 UT, 9 March 1998: (a) shows magnetic field magnitude (solid) and the radial component of velocity (dotted) obtained integrating $22 \mathrm{keV}$ ions (energy interval between about 20 and $25 \mathrm{keV}$ ). (b) shows flux of $22 \mathrm{keV}$ ions in outward (solid) and inward (dotted) directions, units are arbitrarily. (c) shows flux of $22 \mathrm{keV}$ ions in eastward (solid) and westward (dotted) directions, units are arbitrary.

total pressure can become almost constant when we take into account all energetic particles. We have not taken into account the pressure of electrons that contribute about 1/5-1/7 of the total pressure (Baumjohann et al., 1989).

\subsection{Phase velocity, the 9 March 1998 event}

Haerendel et al. (1999) estimated the azimuthal phase velocity of compressional pulsations using close conjunction of Equator-S and Geotail satellites for the 9 March 1998 event. The obtained velocity was about $20 \mathrm{~km} / \mathrm{s}$ eastward for the time period around the conjunction, 1100-1300 UT. During the same time period, the average azimuthal plasma velocity is $21 \mathrm{~km} / \mathrm{s}$ from the Equator-S data and $16 \mathrm{~km} / \mathrm{s}$ from the Geotail data (if not otherwise specified, we use the term plasma velocity as the velocity obtained integrating ions in the energy interval $32 \mathrm{eV}-40 \mathrm{keV}$ ). This indicates that the compressional pulsations move with a phase velocity that is close to plasma velocity.

On 9 March 1998 the phase velocity of compressional pulsations is approximately equal to the plasma velocity, also at time intervals outside the satellite conjunction. To show this we estimate the phase velocity using the finite gyroradius method (Su et al., 1977). This method uses the phase difference between ion flux measurements from opposite directions to estimate the phase velocity of the passing wave. In Fig. 7 we show such estimates for a few compressional variations in the magnetic field during the time period 1500 1540 UT. The solid line in Fig. 7a shows the magnetic field 
magnitude. A cross-correlation analysis between the outward and inward flux of $22 \mathrm{keV}$ ions, shown in Fig. 7b, gives a time shift of $75 \mathrm{~s}$, such that the phase velocity is eastward. This corresponds to a phase velocity of about $20 \mathrm{~km} / \mathrm{s}$ eastward (assuming that the magnetic field magnitude is $28 \mathrm{nT}$ ). The cross-correlation between eastward and westward ion flux, shown in Fig. 7c, gives a time shift of -12 s. This indicates that the phase velocity has a small outward component of about $3 \mathrm{~km} / \mathrm{s}$. However, one should note that the error in the radial phase velocity is of the same order as the radial phase velocity itself; we cannot fix the direction of the ion flux measurements with a precision better than some 10 degrees.

Thus, from the finite gyroradius method, we obtain an eastward phase velocity of about $20 \mathrm{~km} / \mathrm{s}$. During the same period, the mean value of the eastward component of the plasma velocity is $19 \mathrm{~km} / \mathrm{s}$. The estimates of both the phase velocity and the plasma velocity have an uncertainty of about $2-5 \mathrm{~km} / \mathrm{s}$. We again see that the compressional pulsations move approximately with same phase velocity as plasma velocity (note that the plasma velocity is a sum of the $\boldsymbol{E} \times \boldsymbol{B}$ drift and diamagnetization drifts). We obtain a similar agreement for other time intervals during 9 March 1998. For example, during the time interval 1540-1640 UT, the phase velocity is about $16 \mathrm{~km} / \mathrm{s}$, while the eastward component of the plasma velocity is about $13 \mathrm{~km} / \mathrm{s}$. During the time interval 1640-1720 UT, the phase velocity is about $6 \mathrm{~km} / \mathrm{s}$ and the plasma velocity is about $3 \mathrm{~km} / \mathrm{s}$. In all cases, the radial component of the phase velocity is much smaller than the azimuthal component.

\subsection{Phase velocity, the 25 April 1998 event}

The phase velocity obtained from the conjunction of satellites is about $30 \mathrm{~km} / \mathrm{s}$ eastward. The phase velocity obtained using the finite gyroradius method of $30 \mathrm{keV}$ ions is about $25-30 \mathrm{~km} / \mathrm{s}$ eastward, while the finite gyroradius method also shows that the radial component of the phase velocity is small in comparison to the azimuthal component. Thus, both methods to estimate phase velocity indicate that pulsations propagate eastward, and there is a reasonable agreement in velocity amplitude.

Plasma velocity calculations are more difficult because a large part of the ions are outside the energy range of particle instruments. The average azimuthal velocity measured by Geotail is $20 \mathrm{~km} / \mathrm{s}$ (about $25 \mathrm{~km} / \mathrm{s}$ at the beginning of event and $15 \mathrm{~km} / \mathrm{s}$ at the end). However, the average plasma velocity measured by Equator-S is only about $0 \mathrm{~km} / \mathrm{s}$. The explanation for this discrepancy can be diamagnetic drifts. We can make a rough estimate of the diamagnetic drifts. In the distance of 3.5 $R_{E}$ between satellites, the radial component of magnetic field changes from about $-30 \mathrm{nT}$ to $40 \mathrm{nT}$ (magnetic field during this event is very tail-like). The Earth's internal field makes up approximately $20 \mathrm{nT}$ of this change. If we assume that the rest is caused by a local current sheet and that the current is carried by ions (in the $\boldsymbol{E} \times \boldsymbol{B}$ frame of reference), we obtain a diamagnetic drift of the order of $15 \mathrm{~km} / \mathrm{s}$ (ion density is about $0.7 \mathrm{~cm}^{3}$ during this event). Since Geotail is further away from the magnetic equator, it can already be in a region outside the current sheet. Then the contribution from the diamagnetic drift would be seen primarily by Equator-S. This can explain the discrepancy of azimuthal plasma velocities measured by both satellites.

An additional support for this explanation is that if in Equator-S data we integrate only low energy ions (below 2 $\mathrm{keV}$ ), thus measuring a cold component which does not contribute to diamagnetization drifts, we obtain azimuthal velocities of the order of $20 \mathrm{~km} / \mathrm{s}$ eastward. This is close to the velocity measured by Geotail and also close to the phase velocity of compressional pulsations. Since the velocity of cold ions is dominated by the $\boldsymbol{E} \times \boldsymbol{B}$ drift, this can indicate that pulsations move approximately with $\boldsymbol{E} \times \boldsymbol{B}$ velocity and not with the plasma velocity. However, a large part of the ions were outside the energy range of particle instruments and therefore, definite statements are not possible.

\subsection{Diamagnetization drifts}

The role of diamagnetization drifts is also important when analyzing the structure of compressional pulsations. Above, we have shown that compressional pulsations correspond to regions of high and low magnetic field drifting azimuthally past the satellite. Since pulsations are almost pressure balanced, we expect that currents between regions of different magnetic field magnitudes will be carried as a diamagnetization drift of ions. From the finite gyroradius method, we know that variations in the magnetic field magnitude are largest in the azimuthal direction. Therefore, we expect that the diamagnetization drifts will be the largest in the meridional plane perpendicular to the direction of the magnetic field. We will take this direction for the radial direction because during our event, it is close to the true radial direction. In this radial direction, the total plasma velocity can be even dominated by the diamagnetization drift component. This we demonstrate in Fig. 8.

Figure 8a shows the cross-sections of the proton distribution function for the time period 1500-1540 UT, 9 March 1998 (the same as in Fig. 7). Figure 8b-d show the magnetic field magnitude (solid) and the radial velocity components (dotted) obtained by integrating different energy ranges of the proton distribution function. Due to the difficulties related to the particle instrument calibration for directions parallel to the satellite axis (which, in our case, coincides with the radial direction), there can be a bias in the zero-line of velocities. The diamagnetization drift is carried primarily by high energy particles and therefore, best seen in Fig. 8c; the maxima of the radial velocity are correlated with the periods of decrease in the magnetic field magnitude (as expected for eastward propagation). On other hand, the radial velocity obtained integrating low velocity ions, shown in Fig. 8b, which we expect to be dominated by $\boldsymbol{E} \times \boldsymbol{B}$ drift, does not show any correlation. The radial component of the total plasma velocity, shown in Fig. 8d, is well correlated with the velocities of high energy protons, as seen in Fig. 8c. From this we can 

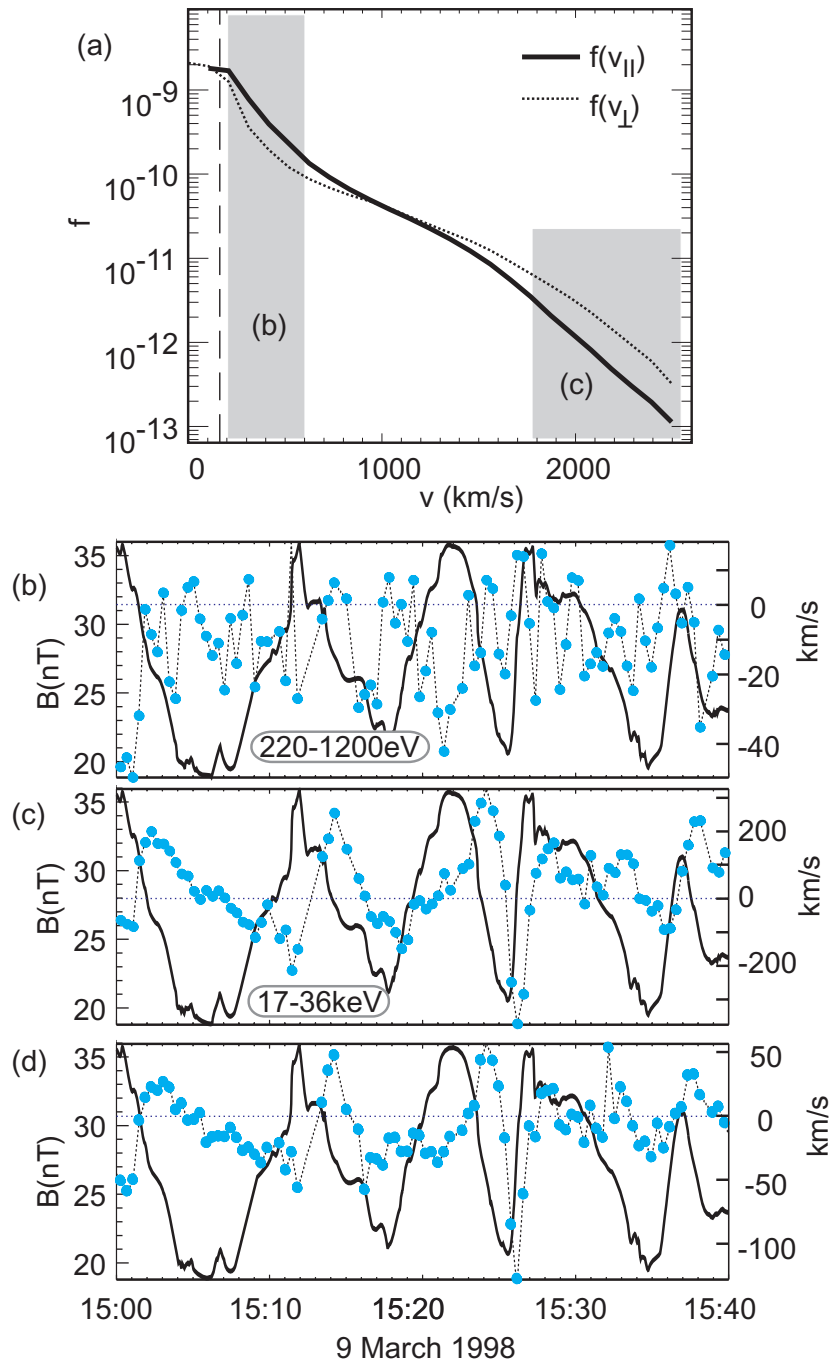

Fig. 8. (a) The cross-section of distribution function for period 1500-1540 UT, 9 March 1998. Velocity intervals which are integrated to obtain results in panels (b) and (c) are marked gray. (b) The magnetic field magnitude (solid) and the radial component of plasma velocity (dotted), integrating ions in the energy interval 140-960 eV. (c) The same as (b) only for energy interval 5-36 keV. (d) The same as (b) only for the whole instrument energy interval, $32 \mathrm{eV}-36 \mathrm{keV}$.

conclude that the radial component of the total plasma velocity is dominated by the diamagnetization drifts. This is also consistent with the rough velocity estimates from Ampere's law. A typical magnetic field gradient of $10 \mathrm{nT} / 2000 \mathrm{~km}$ and plasma density of $0.8 \mathrm{~cm}^{-3}$ corresponds to plasma velocity (which is the same as a diamagnetic drift because of the pressure balance) of the order of $30 \mathrm{~km} / \mathrm{s}$. This velocity is about the same as the velocity seen in Fig. 8d.

If the radial velocity component is dominated by diamagnetization drift, then we expect that on the afternoon side, the phase shift between the component of plasma velocity and the magnetic field magnitude will be opposite to that on the morning side. In the afternoon sector, maxima in the radial velocity will coincide with an increase in the magnetic

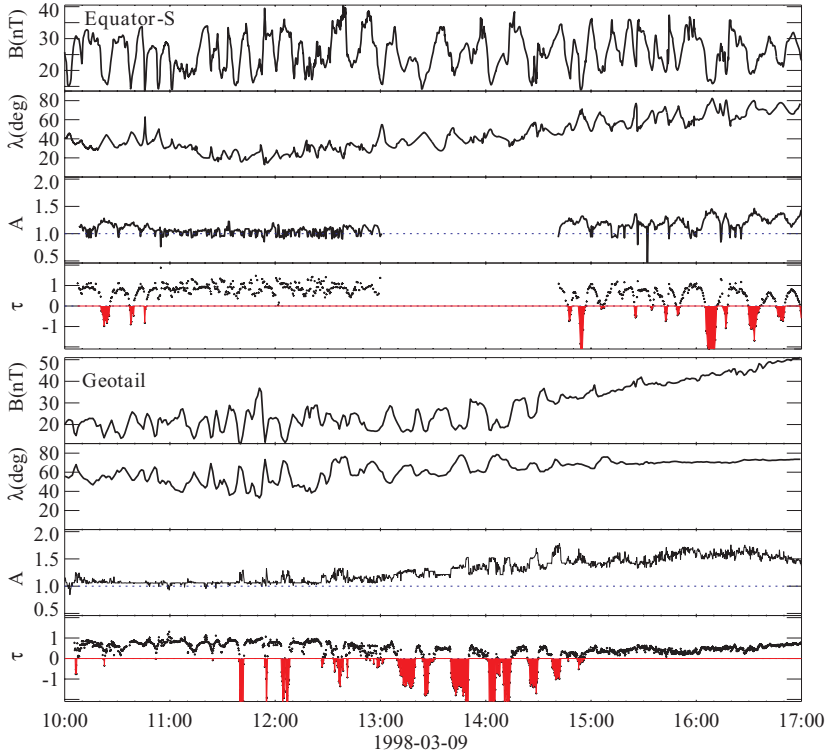

Fig. 9. Figure shows for Equator-S and Geotail: magnetic field magnitude $B$, angle between the direction of magnetic field and the magnetic equator $\lambda$ (90 means perpendicular), temperature anisotropy $A=T_{\perp} / T_{\|}$, and mirror instability condition $\tau=$ $1+\beta_{\perp}\left(1-T_{\perp} / T_{\|}\right)$(instability condition is satisfied where $\tau<0$, colored gray).

field magnitude (magnetic field lags the radial velocity component). Exactly this type of phase relationship has been observed by Geotail (A. Nishida, Spring AGU 1999).

\subsection{Pulsation generation}

In many studies it has been concluded that compressional Pc5 waves are most likely drift mirror-type waves, where it is important to take into account the coupling of compressional and shear components in a curved magnetic field. There are also suggestions about the compressional waves being magnetospheric cavity waves or surface waves generated by the Kelvin Helmholtz instability. However, there is not much data supporting the latter suggestions. We cannot exclude that several generation mechanisms of compressional pulsations act at different regions and/or at different times.

The condition of the drift-mirror instability in a straight field line geometry, for the case $k_{\|} \ll k_{\perp}$, has been found to be $\tau=1+\beta_{\perp}\left(1-T_{\perp} / T_{\|}\right)<0$ (Hasegawa, 1969). This condition requires that the plasma anisotropy is high enough to generate waves, $T_{\perp} / T_{\|}>1+1 / \beta_{\perp}$. For waves with $k_{\|} \approx k_{\perp}$, the instability requires even higher anisotropy. On the other hand, magnetic field curvature changes the instability character (ballooning terms should be taken into account), and decreases the anisotropy required for the instability (Chen and Hasegawa, 1991; Cheng and Qian, 1994). It has been shown that for large curvature, an instability can develop even if the plasma is isotropic. One should also remember that it is not the local instability condition that should be satisfied, but the integral value along the field line. 
For the 9 March 1998 event, Fig. 9 shows the magnetic field magnitude, the angle $\lambda$ between the direction of magnetic field and the magnetic equator (in SM coordinates), the temperature anisotropy $A=T_{\perp} / T_{\|}$, and the mirror instability condition $\tau$. It has been shown before (Zhu and Kivelson, 1994) that when compressional pulsations are present, the mirror instability condition tends to be satisfied in the regions close to the magnetic equator. Our data are partially consistent with these observations. We can use the angle $\lambda$ as an approximate indicator of how far satellites are from the real magnetic equator. During 9 March 1998, Equator-S is closest to the equator at the beginning and at the end of the interval, and these are periods of time when the mirror condition is satisfied. Geotail is close to the equator during the whole time period and a mirror instability condition is often satisfied. One can also see that for Geotail, the mirror instability condition tends to be better satisfied just before Geotail enters from the plasma sheet into the inner magnetosphere (around 1500 UT). In comparison to Equator-S, Geotail is moving relatively fast in the radial direction and therefore, Geotail data can also reflect the radial structure of the region, i.e. the mirror instability condition is better satisfied both close to the equator and close to the inner edge of the plasma sheet.

Figure 9 also shows that the mirror instability condition is not continuously satisfied, but only in regions where compressional pulsations have a minimum in the magnetic field. In these regions, not only is $\beta$ usually much larger than one, but also plasma anisotropy is higher. This can be an indication of the instability saturation.

During the 25 April 1998 event, both satellites show that perpendicular and parallel temperatures are approximately the same. In this case, the simplified instability condition of the mirror mode cannot be satisfied (therefore, we do not plot data for this event). There still can be several explanations for wave generation: the anisotropy can be in high energy particles that are not measured (above $40 \mathrm{keV}$ ), the mirror condition can be satisfied closer to the magnetic equator or closer to the inner magnetosphere, or the radial pressure gradient and/or magnetic field curvature can be high enough that the ballooning-mirror modes can grow even in the isotropic plasma. From this event we cannot distinguish which is the case. However, the high curvature of the magnetic field (of the order of $2 R_{E}$ ), small variations in the SM-Z component and the eventual absence of the anisotropy can indicate that ballooning effects dominate the wave generation; these conditions are predicted by the drift ballooning-mirror instability (Cheng and Qian, 1994). Due to the high curvature, only a small pressure gradient would be necessary to initiate the instability, even in isotropic plasma.

\section{Results and discussion}

We have analyzed two conjunction events, 9 March 1998 and 25 April 1998, between Equator-S and Geotail, during which satellites observe compressional Pc5 pulsations with eastward phase velocities. The 9 March event was observed at 5.0-6.5 MLT during calm geomagnetic conditions, while the 25 April event was observed at 4.0-4.3 MLT during a geomagnetically active period a few hours after a substorm.

Satellite data show that the phase velocity of the compressional pulsations is eastward (sunward) and has values almost equal to the local plasma velocity for the 9 March 1998 event. Similar values of phase velocities are obtained using data from two satellites in conjunction or using the finite gyroradius method. In comparing the phase velocity with the plasma velocity, it is interesting to know how large a contribution to the plasma velocity comes from $\boldsymbol{E} \times \boldsymbol{B}$ drift and diamagnetic drifts. We can assume that the radial scale of the changes in the background pressure is larger than the spatial scale of the compressional pulsations (which is of the order of $1 R_{E}$ ). The data of Geotail and Equator-S before and after the conjunction, when there is a radial separation of satellites, support this assumption. This implies that the diamagnetic drift (due to the radial plasma pressure gradient) would be less than $5 \mathrm{~km} / \mathrm{s}$. Therefore, during this event, the azimuthal velocity of plasma, which is of the order of $20 \mathrm{~km} / \mathrm{s}$, is dominated by the $\boldsymbol{E} \times \boldsymbol{B}$ drift. Due to the small values of expected diamagnetization drifts, from the data, we cannot distinguish whether pulsations move with $\boldsymbol{E} \times \boldsymbol{B}$ drift or plasma velocity. Note that the phase velocity of pulsations does not agree with a typical drift velocity of $\mathrm{keV}$ ions that drift with several tens of $\mathrm{km} / \mathrm{s}$ westward in the $\boldsymbol{E} \times \boldsymbol{B}$ reference frame.

The second 25 April 1998 event also shows that compressional pulsations move eastward with a characteristic phase velocity of about $25-30 \mathrm{~km} / \mathrm{s}$ (it varies during the event). Similarly, the data from satellite conjunction give phase velocity values consistent with the values obtained from the finite gyroradius method. In addition, the azimuthal plasma velocity of about $20 \mathrm{~km} / \mathrm{s}$ eastward, measured by Geotail, is relatively close to the phase velocity values. However, the Equator-S shows the azimuthal plasma velocities of about $0 \mathrm{~km} / \mathrm{s}$ that significantly differs from the phase velocity values. We speculate that this occurs because during this event, the magnetic field is tail-like and Equator-S, being closer to the magnetic equator than Geotail, observes the diamagnetic drift creating the current. In support of this, we show that the integration of only low energy ions (below $2 \mathrm{keV}$ ) gives the azimuthal plasma velocity of about $20 \mathrm{~km} / \mathrm{s}$ eastward that is consistent with Geotail data and also close to the phase velocity of pulsations. This would indicate that close to the magnetic equator, pulsations can move eastward in the plasma reference frame; they still can have a small westward component in the frame moving with $\boldsymbol{E} \times \boldsymbol{B}$ velocity. More studies of similar events are necessary to draw definite conclusions.

Periodic structures in $\beta \geq 1$ plasma with the total pressure almost constant are very common in cosmic plasma. They have been seen in the magnetosheath (mirror waves), solar wind (magnetic holes), and at other planets. Our data do not contradict the earlier observations that compressional pulsations are pressure balanced structures, $p_{B}+p_{\perp}=$ const. Pressure balance is clearly seen during the 9 March 1998 event. However, we cannot show the pressure balance for 
the 25 April 1998 event because a large part of the ions have energies above $40 \mathrm{keV}$, which is the limit for ion instruments used to calculate thermal pressures. We can still see that the pressure of ions with energies below $40 \mathrm{keV}$ and the magnetic pressure oscillate in antiphase.

We show that compressional Pc5 waves move with a velocity which is approximately equal to the plasma velocity. A wave mode that has such a low phase velocity and also low frequency is a mirror mode (with appropriate modifications in the case of gradients in magnetic and thermal pressure, and curvature in magnetic field). Here it is important to remark that analyzing pressure changes in the mirror mode we cannot apply fluid description. In a fluid description of homogeneous plasma, assuming that particles travel fast enough, pressure changes in a compressional wave, such as the mirror mode, are given by $\delta p_{\perp}=\left(1-T_{\perp} / T_{\|}\right) \beta_{\perp} \delta p_{B}$. Thus, for the magnetic and thermal pressure to be in antiphase, plasma should be anisotropic, $T_{\perp}>T_{\|}$, and only in special cases, magnetic and thermal pressures should balance each other. However, Southwood and Kivelson (1993) show that if the contribution from resonant particles is taken into account during the growth of the mirror mode, magnetic and thermal pressures balance each other. A similar result was obtained earlier by Southwood (1976). We can expect that the same will hold for the nonlinear saturation phase of the mirror mode, also including effects of gradients and magnetic field curvature. Then mirror structures will be pressure balanced and will not satisfy the pressure variations predicted from the fluid theory.

We have shown that in our event compressional pulsations have phase velocities sunward, which is opposite to the magnetosheath velocity. Therefore, Kelvin-Helmholtz at the magnetopause is most probably not involved in their generation. Even though we cannot exclude a possibility of the Kelvin Helmholtz instability at the inner boundary of the sunward convection region (the region of region 2 currents), we do not see large velocity shear there. To interpret the observed waves as cavity or wave-guide modes, there should be an explanation for the pressure balance, antisymmetric structure, sunward phase velocity and high- $m$ number, each of which is not a natural consequence of those models.

Acknowledgement. Topical Editor G. Chanteur thanks two referees for their help in evaluating this paper.

\section{References}

Anderson, B. J., Engebretson, M. J., Rounds, S. P., Zanetti, L. J., and Potemra, T. A., A statistical study of Pc 3-5 pulsations observed by the AMPTE/CCE magnetic fields experiment. 1. Occurrence distributions, J. Geophys. Res., 95, 10495-10523, 1990.

Allan, W. and Wright, A. N., Large-m waves generated by small-m field line resonances via the nonlinear Kelvin-Helmholtz instability, J. Geophys. Res., 102, 19927-19933, 1997.

Baumjohann, W., Paschmann, G., and Cattell, C. A., Average plasma properties in the central plasma sheet, J. Geophys. Res., 94, 6597-6606, 1989.
Baumjohann, W., Sckopke, N., LaBelle, J., Klecker, B., Luhr, H., and Glassmeier, K. H., Plasma and field observations of a compressional Pc 5 wave event, J. Geophys. Res., 92, 12203-12212, 1987.

Chen, L. and Hasegawa, A., Kinetic-theory of geomagneticpulsations. 1. Internal excitations by energetic particles, J. Geophys. Res., 96, 1503-1512, 1991.

Chen, L. and Hasegawa, A., Kinetic-theory of geomagneticpulsations. 2. Ion flux modulations by transverse-waves, J. Geophys. Res., 99, 179-182, 1994.

Cheng, C. Z. and Qian, Q., Theory of ballooning-mirror instabilities for anisotropic pressure plasmas in the magnetosphere, J. Geophys. Res., 99, 11193-11209, 1994.

Fornacon, K.-H., Auster, H. U., Georgescu, E., Baumjohann, W., Glaßmeier, K.-H., Rustenbach, J., and Dunlop, M., The magnetic field experiment onboard Equator-S and its scientific possibilities, Ann. Geophysicae, 17, 1521-1527, 1999.

Haerendel, G.,. Baumjohann, W, Georgescu, E., Nakamura, R., Kistler, L. M., Klecker, B., Kucharek, H., Vaivads, A., Mukai, T., Kokubun, S., High-beta plasma blobs in the morningside plasma sheet, Ann. Geophysicae, 17, 1592-1601, 1999.

Hasegawa, A., Drift mirror instability in the magnetosphere, Phys. Fluids, 12, 2642-2650, 1969.

Hedgecock, P. C., Giant Pc 5 pulsations in the outer magnetosphere: A survey of HEOS-1 data, Planet. Space Sci., 24, 921-935, 1976.

Jacobs, J. A., Kato, Y., Matsushita, S., and Troitskaya, V. A., Classification of geomagnetic micropulsations, J. Geophys. Res., 69, 180, 1964.

Khurana, K. K. and Kivelson, M. G., Ultralow frequency MHD waves in Jupiter's middle magnetosphere, J. Geophys. Res., 94, 5241-5254, 1989.

Kistler, L. et. al, Testing electric field models using ion energy spectra from the Equator-S ion composition (ESIC) instrument, Ann. Geophysicae, 17, 1611-1621, 1999.

Kivelson, M. G., Etcheto, J., and Trotignon, J. G., Global compressional oscillations of the terrestrial magnetosphere - the evidence and a model, J. Geophys. Res., 89, 9851-9856, 1984.

Kokubun, S., Yamamoto, T., Acuna, M. H., Hayashi, K., Shiokawa, K., and Kawano, H., The GEOTAIL magnetic field experiment, J. Geomagn. Geoelec., 46, 7-21, 1994.

Lessard, M. R., Hudson, M. K., and Luhr, H., A statistical study of Pc 3-Pc 5 magnetic pulsations observed by the AMPTE/Ion Release Module satellite, J. Geophys. Res., 104, 4523-4538, 1999.

Lin, N., McPherron, R. L., Kivelson, M. G., and Williams, D. J., An unambiguous determination of the propagation of a compressional Pc 5 wave, J. Geophys. Res., 93, 5601-5612, 1988.

Mukai, T., Machida, S., Saito, Y., Hirahara, M., Terasawa, T., Kaya, N., Obara, T., Eijiri, M., and Nishida, A., The low energy particle (LEP) experiment onboard the Geotail satellite, J. Geomagn. Geoelec., 46, 669-692, 1994.

Nakamura, Haerendel, G., Baumjohann, W., Vaivads, A., Kucharek, H., Klecker, B., Georgescu, E., Kistler, L. M., Mukai, T., Kokubun, S., Eglitis, P., Substorm observations in the early morning sector with Equator-S and Geotail, Ann. Geophysicae, 17, 1602-1610, 1999.

Rostoker G. and Samson, J. C., Can substorm expansive phase effects and low frequency Pc magnetic pulsations be attributed to the same source mechanism?, Geophys. Res. Lett., 11, 271-274, 1984.

Sonnerup, B. U. Ö., Cahill, Jr., L. J., and Davis, L. R., Resonant vibration of the magnetosphere observed from Explorer 26, J. Geophys. Res., 74, 2276-2288, 1969. 
Southwood, D. J., A general approach to low-frequency instability in the ring current plasma, J. Geophys. Res., 81, 3340-3348, 1976.

Southwood, D. J. and Kivelson, M. G., Mirror instability. 1. Physical mechanism of linear instability, J. Geophys. Res., 98, 91819187, 1993.

Su, S. Y., Konradi, A., and Fritz, T. A., On propagation direction of ring current proton ULF waves observed by ATS 6 at $6.6 R_{E}$, J. Geophys. Res., 82, 1859-1868, 1977.

Takahashi, K., Cheng, C. Z., McEntire, R. W., and Kistler, L. M., Observation and theory of Pc 5 waves with harmonically related transverse and compressional components, J. Geophys. Res., 95, 977-989, 1990.

Takahashi, K., Higbie, P. R., and Baker, D. N., Azimuthal propagation and frequency characteristic of compressional Pc 5 waves observed at geostationary orbit, J. Geophys. Res., 90, 14731485, 1985.

Takahashi, K., Fennell, J. F., Amata, E., and Higbie, P. R., Fieldaligned structure of the storm time Pc 5 wave of November 1415, 1979, J. Geophys. Res., 92, 5857-5864, 1987.

Takahashi, K., Lopez, R. E., McEntire, R. W., Zanetti, L. J., Kistler, L. M., and Ipavich, F. M., An eastward propagating compressional Pc 5 wave observed by AMPTE/CCE in the postmidnight sector, J. Geophys. Res., 92, 13472-13484, 1987.

Zhu, X. and. Kivelson, M. G, Compressional ULF waves in the outer magnetosphere. 2. A case-study of Pc-5 type wave activity, J. Geophys. Res., 99, 241-252, 1994.

Zhu, X. and Kivelson, M. G., Compressional ULF waves in the outer magnetosphere. 1. Statistical study, J. Geophys. Res., 96, 19451-19467, 1991. 